\title{
In Situ Electrical Characterization of Single Nanofibers Using a Nanomanipulator in an FIB/SEM Microscope
}

\author{
Katherine Roelofs ${ }^{*}$, Shiyou Xu*, Gerald Poirier ${ }^{*}$, Nan Yao* \\ *Princeton Institute for the Science and Technology of Materials, Princeton University, Princeton \\ NJ 08544 USA
}

The purpose of this research is to characterize the electrical properties of individual lead zirconate titanate (PZT) nanofibers and to develop a general procedure for electrically characterizing one-dimensional (1-D) nanostructures with the Zyvex nanomanipulator in a FIB/SEM dual beam microscope.

Piezoelectric PZT nanofibers have potential as the working components of microscale sensing and actuating devices such as mechanical sensors, ultrasonic transducers, structural health monitors, and energy scavenging devices [1,2]. To date, most research on 1-D piezoelectric nanostructures has dealt with $\mathrm{ZnO}$ nanowires and nanobelts, though bulk PZT has higher piezoelectric coefficients than bulk $\mathrm{ZnO}$. Also, previous research on individual 1-D nanostructure properties is relatively sparse, as individual structures are difficult to handle and characterize. The four-probe nanomanipulator, which interfaces with a Kiethley current-voltage characterization system and operates in an FIB/SEM dual beam microscope, has great potential for electrical characterization of 1-D nanostructures. The benefits of this system include the ability to manipulate individual nanostructures, a high vacuum level for precise measurements, immediate visual feedback from the electron beam, the etching and deposition capabilities of the ion beam, and the flexibility of measurement setup using four probes. The difficulties in this system are possible contamination from the ion beam, charging effects from the electron beam, and the question of contact resistance between the probe tips and the nanostructures.

The nanofibers of $\mathrm{PbZr}_{0.52} \mathrm{Ti}_{0.48} \mathrm{O}_{3}$ were synthesized by mixing the PZT precursors with polyvinyl pyrrolidone (PVP), electrospinning fibers onto a silicon substrate, and then annealing the fibers to produce solid PZT with the perovskite phase [3]. On average, the nanofibers characterized had lengths on the order of 20 microns and diameters of $95 \pm 10 \mathrm{~nm}$. The four probes of the nanomanipulator system are linked to a Keithley system which supports a voltage bias of up to $420 \mathrm{~V}$, and current measurement accuracy to 10 nanoAmps. The probes have replaceable tungsten tips which taper to diameters of $200 \mathrm{~nm}$. For current-voltage characterization, an individual nanofiber was lifted from the substrate with a tungsten probe tip. A first platinum contact was made between the nanofiber and the tip, using the metal deposition capabilities of the FIB. Then, a second probe was positioned such that a second platinum contact can be deposited. The nanofiber bridged the two tungsten probes, completing the circuit (Fig. 1). To measure conductivity, the Keithley system applied a voltage bias sweep between the probes and measures the resulting current.

Characterizing five individual PZT nanofibers gave a conductivity of $0.02 \pm 0.03(\mathrm{~S} / \mathrm{cm})$. This is unlikely to be a true measure of a nanofiber's conductivity, as this value is $2 * 10^{5}$ times higher than the bulk conductivity of PZT $\left[10^{-7}(\mathrm{~S} / \mathrm{cm})\right]$. Since the nanofibers were characterized with a two-point measurement setup, it was considered whether a large contact resistance could 
overwhelm measurements of the nanofiber's resistance. However, the nanoscale diameter of the fiber gives it such a high resistance $(20 \mathrm{G} \Omega)$, that typical contact resistances of the order of $1 \mathrm{k} \Omega$ should be insignificant. Another possibility considered is that platinum could have coated the entire wire during contact deposition. This is unlikely, as the incident angle of the ion beam to the length of the nanofiber during deposition is unfavorable to metal accumulation on the wire itself. Finally, $\mathrm{Ga}^{+}$implantation from the ion beam was considered as a source of contamination. Exposing the nanofibers to the ion beam for increasing lengths of time, it was observed that the PZT nanofibers' conductivity increased significantly with ion exposure (Fig. 2). Simulations of damage to PZT nanofibers from $\mathrm{Ga}^{+}$bombardment were conducted using SRIM software to further explore the effect of exposure time and incident angle upon the individual nanofibers. For a $30 \mathrm{kV}$ beam normal to a $100 \mathrm{~nm}$ deep, flat PZT substrate, it was calculated that $4 \%$ of the incident $\mathrm{Ga}^{+}$ions backscatter from the PZT. For a $52^{\circ}$ incident angle, which is the angle between the electron and ion beam in the microscope and a common setting, $14 \%$ of the $\mathrm{Ga}^{+}$ions backscatter (Fig. 3). It is feasible that change in the $\mathrm{Ga}^{+}$dose with incident angles accounts for the experimentally found difference in the rate of conductivity increase with ion exposure for the two different PZT nanofibers.

This paper will outline a successful protocol for using the nanomanipulator system in an FIB/SEM microscope to characterize the electrical properties of individual PZT nanofibers and will discuss quantification of the major sources of experimental error in such measurements.

\section{References}

[1] Z. L. Wang and J. Song, Science. 312 (2006) 242.

[2] J. Lu and Z. Fan, Mater. Sci. Eng. R. 52 (2006) 49-91.

[3] X. Chen, S. Xu, N. Yao, W. Xu, and Y. Shi, Appl. Phys. Lett. 94 (2009) 253113.

[4] This work is supported in part by the National Science Foundation-MRSEC (DMR0819860).

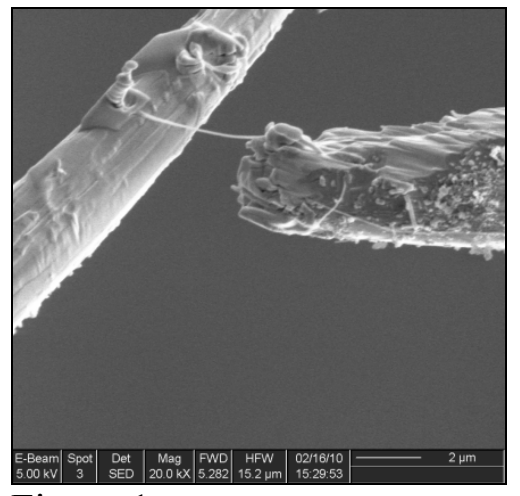

Figure 1.

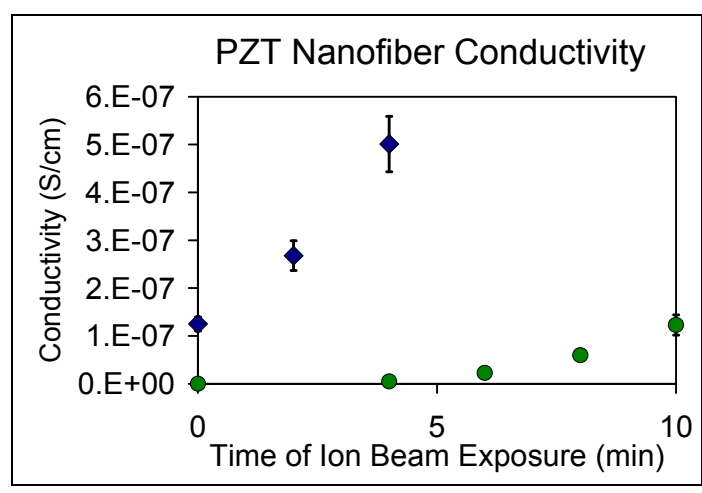

Figure 2.

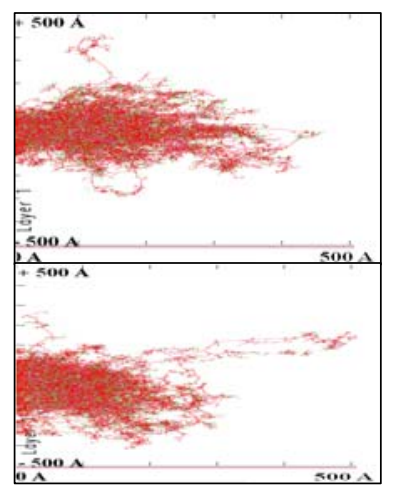

Figure 3.

FIG. 1. SEM image of experimental setup for electronic characterization of an individual PZT nanofiber.

FIG. 2. Increase in conductivity of PZT nanofibers upon exposure to ion beam with acceleration voltage of $30 \mathrm{kV}$ and current of $10 \mathrm{pA}$.

FIG. 3. Cross sections of the interaction volume of $500 \mathrm{Ga}^{+}$ions with $30 \mathrm{keV}$ energy onto a PZT target for $\alpha=0^{\circ}$ (top) and $52^{\circ}$ (bottom), as simulated using the SRIM software. 